\title{
The Microbiota of a Mite Prey-predator System on Different Host Plants Are Characterized by Functional Redundancy and Dysbiosis
}

\section{Bruna Laís Merlin ( $\boldsymbol{\sim}$ bruna.merlin@usp.br)}

University of Sao Paulo Luiz de Queiroz College of Agriculture: Universidade de Sao Paulo Escola Superior de Agricultura Luiz de Queiroz https://orcid.org/0000-0003-4444-3906

\section{Gilberto J Moraes}

University of Sao Paulo Luiz de Queiroz College of Agriculture: Universidade de Sao Paulo Escola Superior de Agricultura Luiz de Queiroz and CNPq researcher

\section{Fernando L Cônsoli}

University of Sao Paulo Luiz de Queiroz College of Agriculture: Universidade de Sao Paulo Escola Superior de Agricultura Luiz de Queiroz

\section{Research Article}

Keywords: Mite symbiosis, Microbial ecology, Mite bacterial diversity, Mite microbiome, Trophic effects on symbiosis

Posted Date: December 3rd, 2021

DOI: https://doi.org/10.21203/rs.3.rs-1128974/v1

License: (c) (1) This work is licensed under a Creative Commons Attribution 4.0 International License. Read Full License 


\section{Abstract}

Microbiota have diverse roles in the life cycles of their hosts, affecting their growth, development, behavior, and reproduction. Changes in physiological conditions of the host can also impact the assemblage of host-associated microorganisms. However, little is known of the effects of host plantprey-predatory mite interactions on mite microbiota. We compared the microbial communities of eggs and adult females of the two sspotted spider mite Tetranychus urticae Koch (Acari: Tetranychidae) and of adult females of the predatory mite Neoseiulus californicus (McGregor) (Acari: Phytoseiidae) on four different host plants (cotton, maize, pinto bean, and tomato) by metabarcoding sequencing of the V3-V4 region of the $16 \mathrm{~S}$ ribosomal RNA gene (16S rRNA), using the Illumina MiSeq platform. Only the egg microbiota of $T$. urticae was affected by the host plant. The microbiota of the predatory mite $N$. californicus was very different from that of its prey, and the predator microbiota was unaffected by the different host plant-prey systems tested. Only the microbiota of the eggs of T. urticae carried Serratia as a high fidelity-biomarker. Biomarker bacteria were also detected in the microbiota of adult females of $T$. urticae and $N$. californicus, with different biomarkers in each host-plant species. The microbiota associated with eggs and adult females of $T$. urticae and adult females of $N$. californicus differed in their potential contributions to the host mite.

\section{Introduction}

Arthropods establish a diverse array of interactions with microorganisms, ranging from mutually beneficial (mutualism) to neutral (commensalism) or parasitic (parasitism) [1-3]. The microorganisms harbored within the body of arthropods include gut microbes and extracellular and intracellular symbionts. The host-microbe relationships can be obligatory or facultative depending on the level of codependence developed [4]. Obligate endosymbionts can provide hosts with essential nutrients, while facultative symbionts contribute to host resistance to natural enemies and alter the range of suitable food sources accessed by their hosts [5].

The intimate relationship between hosts and obligate endocytobionts has selected for more-cooperative symbionts and more-dependent hosts, leading to the reduction of bacterial genomes, as shown for the aphid bacterium Buchnera aphidicola (Gammaproteobacteria, Erwiniaceae). Buchnera has lost genes for non-essential amino acids, cell-surface components, gene regulation, and cell defense, while genes responsible for biosynthesis of amino acids that are essential for aphid hosts have been retained $[5,6]$. Some facultative endosymbionts that manipulate host reproduction or sex determination [7] also affect several fitness traits of their hosts, including their behavior and immune response against natural enemies [8-10].

Free-living symbionts in the gut of arthropods are predicted to play a major role in host-plant adaptation, mainly because they inhabit the main food-processing environment and can respond relatively rapidly to induced changes or to the presence of phytotoxins in the host diet $[11,12]$. The gut bacteria of insects have proven to aid hosts to degrade synthetic pesticides [13-15] and to circumvent host-plant protease 
inhibitors [16], affecting the efficacy of these xenobiotics for insect control. Sharing the luminal space of the gut by a multispecies microbiota that is frequently exposed to new bacteria obtained from the environment also facilitates the acquisition of new genes through exchange of genetic material, which can potentially lead to the development of phenotypes with diverse metabolic profiles [12]. The maintenance of diverse facultative symbionts in host populations is thought to hinge mainly on the balance of selection between the costs and benefits of infection, and is subjected to different selection pressures, such as diet, environmental habitat conditions, host developmental stage, and social behavior [17-21].

The role of bacterial symbionts in the life of animal species drives the routes through which microorganisms are transmitted to next generations [5]. Obligate symbionts are usually transmitted vertically, often through transovarial transmission, while gut bacteria and facultative symbionts can be transmitted vertically or horizontally $[4,5]$. In the case of horizontal transmission, the acquired microbes increase in density after each generation if there are fitness benefits to the host. Moreover, mechanisms that manipulate hosts or promote transmission fidelity might also promote associations and lead to speciation, affecting mate choice [22] or causing hybrid lethality [23].

The host microbiota can also be acquired de novo from species occupying different trophic levels. Predatory mites were reported to share $15 \%$ of their core bacterial taxa with those of the prey [24]. Nevertheless, some of the bacteria acquired from the prey can engage in pathogenic associations with predators, and affect fecundity, longevity, and response to plant volatiles of the new host. These negative effects on the fitness traits of predatory mites can lead to ineffective control of spider mites [25]. Praying mantids, on the other hand, harbor a gut microbiota distinct from that of the host, suggesting that their gut microbiota has a distinct phylogenetic profile from the prey microbiota [26]. Understanding the composition of the bacterial microbiota of predator and prey species targeted for control or used in massrearing systems allows detection of pathogenic bacteria in mass-rearing systems and provides opportunities for manipulation of the bacterial community to improve the health of predatory mites and their performance as biocontrol agents [24].

The two-spotted spider mite Tetranychus urticae Koch (Acari: Tetranychidae) is an important pest of a wide range of greenhouse and field crops around the world, feeding on over 1,100 plant species [27]. Because of the mite's high capacity to evolve resistance to synthetic organic acaricides, the use of biological control of T. urticae with predatory mites, such as Neoseiulus californicus (McGregor) (Acari: Phytoseiidae) is a management strategy adopted worldwide [28]. However, little is known about the effects of host plant-prey-predatory mite interactions on the ecology of the microbiota of prey and predators on different host-plant systems. Here, we tested the hypothesis of vertical transmission of the microbiota from the prey to the predator on different host plants and analyzed the potential functional contributions of the associated microbiota. We used metabarcoding analysis of the V3-V4 region of the 16S rRNA gene to assess the composition of the microbiota of adult females and eggs of the two $₫$ spotted spider mite $T$. urticae and adult females of the predatory mite $N$. californicus reared on four different host plants. 


\section{Material And Methods}

\section{Colonies of phytophagous and predatory mites}

A stock colony of $T$. urticae was maintained under controlled conditions $\left(25 \pm 2^{\circ} \mathrm{C}, 60 \%\right.$ R.H., $14 \mathrm{~h}$ photophase) on leaves of jack bean (Canavalia ensiformis L.). Adult females of T. urticae were transferred from the stock colony to four different host plants: i) cotton (Gossypium hirsutum L.); ii) maize (Zea mays L.); iii) pinto bean (Phaseolus vulgaris L.); and iv) tomato (Solanum lycopersicum L.). Each colony of $T$. urticae was divided into two sub-colonies: i) one to serve as a stock colony of the two囚spotted mite on each host plant, and ii) another to serve as a trophic system in which the predatory mite $N$. californicus was included.

Assessing the microbiota of T. urticae and N. californicus Sampling and DNA extraction

After two generations on different host plants and on each host plant-prey system, samples of eggs and adult females of $T$. urticae and adult females of $N$. californicus (respectively $30,15,15$ /replicate/treatment) were collected from each colony and subjected to genomic DNA (gDNA) extraction, following Gilbert et al. [29]. The DNA extracted was quantified in a spectrophotometer (Biomate 3, Thermo Electron Corp., Madison, WI) and stored at $-20^{\circ} \mathrm{C}$ until further use. Samples were subjected to RNAse A $\left(\right.$ Thermo Scientific ${ }^{\top M}$ ) treatment to remove any residual RNA, following the manufacturer's recommendations.

\section{Amplification, library construction, and metabarcoding sequencing}

gDNA of eggs and adult females of T. urticae and adult females of $N$. californicus was subjected to PCR amplification of the $\mathrm{V} 3-\mathrm{V} 4$ region of the $16 \mathrm{~S}$ rRNA in a thermocycler programmed at $94^{\circ} \mathrm{C}$ for $2 \mathrm{~min}(1$ cycle); $95^{\circ} \mathrm{C}$ for $45 \mathrm{~s}, 55^{\circ} \mathrm{C}$ for $1 \mathrm{~min}$, and $72^{\circ} \mathrm{C}$ for $1.5 \mathrm{~min}$ (32 cycles); and $68^{\circ} \mathrm{C}$ for $10 \mathrm{~min}$ ( 1 cycle), with a final hold at $4^{\circ} \mathrm{C}$. PCRs were run with $10 \mathrm{ng}$ of gDNA, enzyme buffer $(1 \times), 1.5 \mathrm{mM} \mathrm{MgCl}, 0.2 \mathrm{mM}$ of each dNTP, $0.625 \mathrm{U}$ Taq polymerase (GoTaq ${ }^{\circledR}$ DNA Polymerase), and $0.2 \mu \mathrm{M}$ of each $16 \mathrm{~S}$ Illumina primer in a final volume of $25 \mu \mathrm{L}$. Amplification efficiency was determined using $1.5 \%$ agarose gel electrophoresis, following standard procedures [30]. A second amplification reaction was performed using the commercial product Nextera XT DNA Library Preparation Kit (Illumina) for addition of Illumina indexers and adapters. Amplicons were purified with AMPure XP beads, quantified using standard qPCR, and subjected to library preparation using equal amounts of amplicons. Samples were sequenced according to the paired-end protocol $(2 \times 250 \mathrm{bp})$ in the MiSeq Illumina platform at the Multiusers Center of Agricultural Biotechnology, Luiz de Queiroz College of Agriculture, University of São Paulo.

\section{Microbiome analyses}

Sequence analysis was performed using the dada2 package [31] available for the R environment v.4.0.3 [32]. The reads obtained were subjected to quality filters for removal of residual primers and low-quality 
sequences, following the parameters: reads smaller than $250 \mathrm{bp}$ were discarded (truncLen $=250,250$ ), the maximum number of $N$ allowed in the sequences was $0(\max N=0)$, and the maximum number of errors assumed by the analyses was 2 for forward and reverse reads [ $\max E E=c(2,2)]$. Reads were paired and possible chimeric sequences were removed. Then, sequences were aligned against the sequences available in the Silva138 database [33]. Afterward, sequences were classified as Amplicon Sequence Variants (ASVs), using the method described by Callahan et al. [34], considering $99 \%$ as the limit of similarity. Sequences with fewer than 10 counts and less than $10 \%$ prevalence in samples were discarded. Sequences with fewer than 100 counts were combined as "others" to better represent the data.

The ASVs were subjected to alpha-diversity analysis, using the Shannon diversity index [35]. Rarefaction curves were used to determine the quality of the sampling effort, using the depth limit of the smallest library $(=3,013$ reads). Beta diversity was analyzed using the Jaccard index and weighted and unweighted Unifrac distance metrics [36-38]. The Jaccard and Unifrac distance matrices were used in hierarchical grouping, using the principal coordinates analysis (PCoA) for the beta-diversity analysis in the MicrobiomeAnalyst platform (https://www.microbiomeanalyst.ca/), using the nonparametric KruskalWallis and Permanova tests, both at $p<0.05[39,40]$. Hierarchical clustering was also used to produce heatmaps for data visualization, using Pearson as the distance measure and the mean as the clustering algorithm. Differential abundance analysis was performed using the EdgeR test [41] combined with the False Discovery Rate (FDR) method $(p<0.05)$. The Linear Discriminant Analysis Effect Size (LEfSe) method was performed using the nonparametric factorial Kruskal $₫$ Wallis rank sum test to detect features with significant differential abundance, followed by the Linear Discriminant Analysis (LDA) to estimate the effect size of each differentially abundant feature [42].

ASV sequences and abundances were processed using Piphillin (https://piphillin.secondgenome.com/) $[43,44]$ to predict the functional contribution of each microbiota and allow identification of biomarkers. Comparative analyses of the predicted functions for each microbiota obtained in the Piphillin output were also conducted in the MicrobiomeAnalyst platform [39, 40], using EdgeR [41] combined with the False Discovery Rate (FDR) method $(p<0.05)$.

\section{Results}

\section{Data pre-processing}

The metabarcoding sequencing of the 16S rDNA of the microbiota associated with eggs and adult females of $T$. urticae and with adult females of $N$. californicus yielded a total of 2,902,789 reads, with a mean of 80,633 reads per sample. After quality filtering, the total number of paired reads was 591,495 with a mean length of $454 \mathrm{bp}$. Rarefaction curves demonstrated that the sampling efforts were adequate to fully represent the richness of the communities analyzed, as no increase in ASV diversity was observed or unique ASVs detected after sampling 3,013 sequences (Fig. S1).

\section{Taxonomic profiles}


The host plants did not affect the relative abundance of the microbiota associated with eggs and adult females of $T$. urticae and adult females of $N$. californicus at the phylum level. In the microbiota of $T$. urticae, Proteobacteria comprised nearly $99 \%$ of all bacteria detected, with Firmicutes and Bacteroidota comprising less than $1 \%$ of ASVs. Proteobacteria was also the most prevalent $(>85 \%$ of all ASVs) in N. californicus, with a higher relative abundance of Firmicutes, mainly in samples from maize and tomato, and Bacteroidota in samples from cotton and pinto bean (Fig. S2).

Proteobacteria was represented only by Alphaproteobacteria and Gammaproteobacteria.

Alphaproteobacteria comprised more than $90 \%$ of the total microbiota associated with adult females of T. urticae, except for the microbiota of adults from cotton, in which the abundance of Gammaproteobacteria increased to around 35\% of the total ASVs detected (Fig. 1a). The microbiota of eggs of T. urticae was dominated by Gammaproteobacteria, which comprised more than $95 \%$ of the relative abundance of all classes of bacteria detected, regardless of the host plant (Fig. 1a).

Gammaproteobacteria was also the most prevalent in adult females of $N$. californicus, but with a lower relative abundance than observed in eggs of $T$. urticae. Alphaproteobacteria predominated in the microbiota of $N$. californicus from maize, while Gammaproteobacteria predominated in the microbiota of predatory mites from cotton (Fig. 1a). Firmicutes was consistently recorded in samples of predatory mites from maize and tomato, but only sporadically represented in samples of predatory mites from cotton and pinto bean (Fig. 1a).

Serratia (Gammaproteobacteria, Enterobacteriaceae) dominated the microbiota of $T$. urticae eggs from all host plants (Fig. 1b). In contrast to eggs, the microbiota of adult females of $T$. urticae was dominated by the alphaproteobacterium Rickettsia (Rickettsiaceae), comprising more than $75 \%$ of the relative bacterial diversity (Fig. 1b). The prevalence of Rickettsia in the microbiota associated with T. urticaefemales was shared only with Pseudomonas (Gammaproteobacteria, Pseudomonadaceae) in cotton and with Wolbachia (Alphaproteobacteria, Anaplasmataceae) in tomato (Fig. 1b). The microbiota associated with adult females of N. californicus was more diversified, but Serratia, Rickettsia, and Pseudomonas were the most abundant bacteria, changing in relative abundance from host plant to host plant (Fig. 1b). Several other genera were detected in N. californicus females, including Paenibacillus (Bacilli, Paenibacillaceae), common in the microbiota of this mite from tomato (Fig. 1b).

\section{Alpha and beta diversities}

The alpha-diversity analysis indicated that host plants did not affect the microbial community diversity associated with eggs and adult females of $T$. urticae or with adults of $N$. californicus, except for the microbiota associated with $T$. urticae females on pinto bean (Shannon index values: $F=6.05 ; p=0.04$ ). The alpha-diversity of the microbiota associated with eggs of $T$. urticae on pinto bean was also lower than the diversity observed in adults of $N$. californicus (Shannon index values: $F=\otimes 4.36 ; p=0.0471$ ) (Fig. 2). Other comparisons did not result in significant differences for alpha diversity (Table S1).

PCoA analysis using Jaccard indices indicated that the microbiota of T. urticae from maize, pinto bean and tomato were closely clustered, while the microbiota from cotton was more dispersed (Fig. 3a; 3b). 
PCoA analysis indicated no differences in the beta diversity of the microbiota of $N$. californicus in the host plant-prey systems evaluated (Fig. 3c). PCoA analysis using the distances based on weighted Unifrac showed that the microbiota associated with adult females of $T$. urticae from cotton differed most from the others (Fig. 3d), while samples from eggs of T. urticae were grouped (Fig. 3e), and samples from N. californicus were dispersed (Fig. 3f). PCoA analyses based on the unweighted Unifrac index separated the microbiota of adult females of T. urticae from maize and tomato into one group, and those from cotton and pinto bean in another group (Fig. 3g). However, the microbiota of eggs of T. urticae (Fig. $3 \mathrm{~h}$ ) and the predator $N$. californicus (Fig. $3 \mathrm{i}$ ) clustered in accordance with the host plants.

\section{Clustering analysis}

Differences in ASV counts between species (T. urticae and N. californicus) and stages of development of T. urticae (eggs and adult females) contributed more in the clustering analysis than the differences in ASV counts among the host plants (cotton, maize, pinto bean, and tomato) (Fig. 4a). The members of Proteobacteria were over-represented in the microbiota of $T$. urticae compared to the microbiota associated with the predatory mite N. californicus, with Gammaproteobacteria predominating in eggs, and Alphaproteobacteria in adult females of T. urticae (Fig. 4b). The most abundant ASVs of the microbiota of $N$. californicus belonged to Bacilli and Bacteroidia, which were among the least represented ASVs in eggs and adult females of T. urticae.

Serratia was the most abundant ASV in the microbiota of eggs of T. urticae, but showed low abundances in the microbiota of adult females of T. urticae and N. californicus (Fig. 4c). Rickettsia was the most abundant ASV in adult females of T. urticae, followed by Wolbachia, with the exception of the microbiota of $T$. urticae reared on cotton and maize, in which Wolbachia had low abundances. Pseudomonas also appeared in high numbers in the microbiota of adult females of $T$. urticae, but was abundant only in mites reared on cotton plants (Fig. 4c).

The abundance of ASVs belonging to the microbiota associated with the predatory mite varied according to the host plant. Pseudomonas and Flavobacterium were the most abundant ASVs in N. californicus from cotton and pinto bean, while Enterococcus, Bacillus, and Paenibacillus were the most abundant in maize, and Bacillus and Paenibacillus in predatory mites reared on prey from tomato plants (Fig. 4c). Stenotrophomonas was also an abundant ASV in the microbial community associated with females of $N$. califonrnicus from cotton, pinto bean, and tomato (Fig. 4c).

The dendrograms produced using the distances based on weighted Unifrac showed that all samples of $T$. urticae eggs clustered in a clade separated from adult females of T. urticae (Fig. $5 \mathrm{a}$ ) and N. californicus (Fig. 5b), except for one replicate of $T$. urticae eggs from cotton. This replicate clustered with one replicate of $N$. californicus from cotton (Fig. 5a) and two replicates of $T$. urticae eggs from cotton and tomato (Figure $5 b$ ). Adult females of $T$. urticae clustered according to the host plants, except for cotton leaves, while eggs of $T$. urticae and adult females of $N$. californicus did not cluster according to the host plants (Fig. 5a, 5b). 


\section{Comparative analysis}

Comparative analysis showed that the microbiota associated with eggs of T. urticae was affected by the rearing host plant, while we detected no evidence that the microbiota associated with the predatory mite $N$. californicus and adult females of $T$. urticae suffered any effect from the host plant. The effect of the host plant on the diversity and richness of the microbiota associated with eggs of $T$. urticae was observed in mites reared on cotton. One unassigned genus was more abundant in the microbiota of eggs from cotton than from the other host plants.

Comparative analysis from maize indicated 4.5 times more Serratia in the microbiota of $T$. urticae eggs than in the microbiota of $N$. californicus females, but the microbiota of $N$. californicus had 4.3-6.7 times more Rickettsia, Methylophilus, and Pseudomonas than the microbiota of T. urticae eggs (Fig. 6). Eggs of T. urticae from pinto bean had 10 times more Serratia and 4 times more Wolbachia than females of $N$. californicus (Fig. 6). The abundance of Serratia and Wolbachia in the microbiota of eggs of T. urticae from tomato was also higher than in the microbiota of $N$. californicus, with Candidatus Hamiltonella showing an opposite trend (Fig. 6).

The microbiota of eggs had higher abundance of Serratia than the microbiota of adult females of $T$. urticae from all host plants (Fig. 7). The microbiota of adult females of T. urticae had from 4.5 to 6.2 times more Rickettsia than the microbiota of eggs from cotton (Fig. 7) and maize (Fig. 7). Wolbachia and Enterococcus were also more abundant in the microbiota of adult females than in the microbiota of eggs of T. urticae from cotton (Fig. 7), while the opposite was observed for the abundance of the ASV that represented an unassigned genus (Fig. 7).

LDA Effect Size (LEfSe) analyses identified biomarkers in the microbiota associated with $T$. urticae and $N$. californicus reared on different host plants. Serratia was identified as a biomarker for the microbiota of eggs of $T$. urticae regardless of the host-plant association, while the microbiota of adult females of $T$. urticae was characterized by Rickettsia in females from cotton and maize, Rickettsia and Wolbachia from pinto bean, and Wolbachia from tomato (Fig. 8). Biomarkers for the microbiota of the predatory mite were identified only when feeding on pinto bean- (Pseudomonas) and tomato-prey systems (Paenibacillus) (Fig. 8).

\section{Functional prediction analysis}

The relative abundance of the pathways predicted from the microbiota associated with eggs and adult females of $T$. urticae and adult females of $N$. californicus was similar regardless of the host-plant association (Fig. 9), except for the lower relative abundance of the amino-acid metabolism (AAM) and xenobiotic biodegradation and metabolism (XBM) pathways. The relative abundance of the AAM and XBM pathways of the microbiota from $T$. urticae females from maize, pinto bean, and tomato was half that in the remaining samples (Fig. 9). 
Comparisons of the potential functional contribution between the microbiota of eggs of twoðspotted spider mite and that of $N$. californicus females from each host plant indicated significant differences in the potential contribution of each microbiota (Table S2). Differences in the functional contribution changed from plant to plant. In cotton, we did not detect differences in the potential functional contribution for the microbiota of the predatory mite $N$. californicus compared to that of eggs of $T$. urticae. However, most comparisons between the microbiota of the predator and prey eggs indicated a higher potential functional contribution of the predator microbiota than the microbiota associated with eggs of T. urticae (Fig. 10). The contribution of the microbiota of eggs of T. urticae exceeded that of the microbiota of the predatory mite only in pathways related to carbohydrate digestion and absorption in maize, pinto bean, and tomato; glycosphingolipid biosynthesis, linoleic acid metabolism, and various types of $\mathrm{N}$-glycan biosynthesis in maize; arabinogalactan biosynthesis in pinto bean; and the calcium signaling and prolactin signaling pathways in maize and pinto bean (Fig. 10).

We also observed differences in the functional contribution of the microbiota between eggs and adult females of T. urticae from all host plants tested (Fig. 10, Table S3). In general, a higher potential functional contribution was observed for microbiota of eggs of $T$. urticae than for that of adult females, particularly on maize, pinto bean, and tomato (Fig. 10). The egg microbiota functional contribution was higher than that of the microbiota of females in all host plants for the phosphotransferase system (PTS), other glycan degradation, various types of $\mathrm{N}$-glycan biosynthesis, arabinogalactan biosynthesis, and glycosphingolipid biosynthesis (Fig. 10). But the opposite was observed on maize, pinto bean, and tomato plants for pathways involved in thermogenesis, zeatin biosynthesis, protein processing in the endoplasmic reticulum, the p53 signaling pathway, and apoptosis (Fig. 10).

\section{Discussion}

The microbiota of eggs and adult females of the two \spotted spider mite $T$. urticae and of adult females of $N$. californicus were very distinctive, and only the egg microbiota was affected by the host plant. The microbiota of the predatory mite $N$. californicus was very different from that of the prey, and the diversity and abundance of microbiota on this mite were unaffected by the different host plant-prey systems tested. Biomarker bacteria were detected for both T. urticae and N. californicus, but only the microbiota of the egg stage of $T$. urticae showed high fidelity with the biomarker bacterium Serratia in the different conditions tested. Biomarker bacteria were detected in the microbiota of adult females of $T$. urticae, but the biomarkers were affected by the host plant used, while the microbiota of $N$. californicus was moderately impacted by the host plant-prey system used, and different biomarkers were detected, but only in tomato- and pinto bean-prey systems. The microbiota associated with eggs and adult females of T. urticae and adult females of $N$. californicus differed in their potential contributions to the host mite. Nevertheless, changes induced by the host plant in the microbiota of T. urticae and N. californicus did not produce communities with changes in their potential functional contributions.

Members of Serratia colonize a variety of niches and can either establish successful mutualistic associations or act as opportunistic pathogens $[45,46]$. Serratia were also reported associated with the 
ovarioles of Gargara genistae (F.) (Hemiptera: Membracidae), which suggests that it is vertically transmitted, playing a role in host metabolic complementation [47]. However, the presence of Serratia on the phylloplane of plants has also been reported, showing that Serratia may colonize plant surfaces without damaging the plant [48]. The prevalence of Serratia in eggs of T. urticae in all host plants tested and their low abundance in T. urticae adult females suggests that the association between Serratia and $T$. urticae is accidental, occurring through contact of the egg with plant surfaces. The high prevalence of Serratia in eggs of $T$. urticae significantly affected the potential functional contribution provided by the associated microbiota. Since we do not believe that eggs of $T$. urticae could harbor such a high abundance of a single bacterial type in the ooplasm, we suggest that the functional contribution of the egg microbiota of $T$. urticae is likely limited. This is also supported by the lack of indication that Serratia are primary symbionts and transferred to eggs by vertical transmission, as the adult microbiota of $T$. urticae is dominated by the Alphaproteobacteria Rickettsia and Wolbachia. These alphaproteobacteria are reported within oocytes and in cells adjacent to the eggs in the ovaries of $T$. urticae, clearly demonstrating a transovarial route of transmission $[49,50]$. Bacteria transmitted transovarially are often present in low densities in host eggs but increase in density as the host grows and develops, as is the case with Wolbachia [51, 52].

Members of Rickettsia are known for their potential pathogenicity to vertebrates [53] but can also benefit invertebrates by increasing host fitness and the proportion of females, enhancing the immune response to pathogens, and increasing the heat-stress tolerance of the host [54,55]. Previous studies showed that a Rickettsia strain associated with Ixodes scapularis Say (Acari: Ixodidae) possessed all the genes required for the synthesis of folate (vitamin B9), which are not present in the host genome. Folate is the most essential component for cell growth, participating in the one『carbon metabolism pathway in nucleotide synthesis and in the methylation of DNA, RNA, proteins, and phospholipids [56]. Although Rickettsia is horizontally transferred to some hosts $[57,58]$, we did not observe any pathological symptoms in our rearing colonies that would suggest Rickettsia would be pathogenic to adult females of T. urticae. We believe that Rickettsia act as a true symbiont of T. urticae because it has been reported to infect the ovaries and eggs of $T$. urticae, with no evidence of a pathological association [49].

Wolbachia can manipulate host reproduction phenotypes in arthropods via cytoplasmic incompatibility, parthenogenesis, male killing, and male feminization [7, 59, 60], but the environment can also influence the frequencies of endosymbionts [18]. Host plants play an important role in shaping the bacterial community of herbivores, which includes facultative bacterial endosymbionts. In a population of Tetranychus truncatus Ehara (Acari: Tetranychidae) from maize, the infection rates of Wolbachia were lower than those from tomato and soybean, showing that endosymbionts associated with T. truncatus are affected by host plants [61]. Our results showed that the relative abundance of Wolbachia in T. urticae increased from eggs to adult females, and that it was affected by the host plant. Host plants can reduce the prevalence of Wolbachia and the fecundity and hatching rates of T. urticae, indicating that host-plant suitability affects the prevalence of Wolbachia [62]. The reduced genome of members of Wolbachia due to the loss of some essential biosynthetic pathways imposes a nutritional burden on their hosts. The competition for key nutritional resources with hosts was shown to negatively impact host performance 
and affect the host-Wolbachia association in low $\llbracket$ quality host-plant sources, leading to Wolbachia elimination, as observed for T. urticae fed on cotton and maize leaves [51, 62].

The predatory mite $N$. californicus harbors a wide diversity of bacteria, with Bacillus, Flavobacterium, Methylophilus, Paenibacillus, Pseudomonas, Rickettsia, Serratia, Stenotrophomonas, and Wolbachia the as main constituents of its microbiota. The presence of some of these bacteria in the microbiota of eggs of T. urticae (Serratia) and adult females of T. urticae (Pseudomonas, Rickettsia, and Wolbachia) suggests that $N$. californicus may acquire them by horizontal transmission. The existence of a shared microbiota among interacting species belonging to different trophic levels suggests that bacteria can undergo horizontal transfer from one species to another through feeding. Horizontal acquisition of bacteria can occur directly through the consumption of host parts harboring microbes or indirectly through shared food sources with other species. However, analyses of a few host-predator associations demonstrated the existence of additional factors that limit the host microbiota from establishing as the predator microbiota, as in some cases the predator microbiota reflects that of the host $[63,64]$, but not in other cases [26].

Among the major constituents of the mite microbiota, Rickettsia, Serratia, and Wolbachia are endocytobionts, while Bacillus, Flavobacterium, Methylophilus, Paenibacillus, Pseudomonas, and Stenotrophomonas are free-living bacteria, commonly reported as associates of plants or the gut of arthropods [65-69]. Several species of Pseudomonas have been shown to benefit plants by alleviating certain stress conditions, and to provide, for example, plant protection [70-72]. Members of Pseudomonas have been investigated as biopesticides for control of mites $[73,74]$ and insects $[75,76]$. Pseudomonas associated with plants that orally infect and kill herbivorous arthropods carry surface glycan decorations to escape the host humoral immune defense [77], killing the hosts by producing molecules with potent insecticidal activity and lytic enzymes (chitinases, phospholipases) (Flury et al. 2017; Vacheron et al. 2019).

Bacillus associated with predatory mite species were shown to aid digestion in mites, but they also inhibited mite population growth, suggesting the establishment of an opportunistic mode of pathogenesis [24, 79]. Flavobacterium and Stenotrophomonas were considered opportunistic pathogenic bacteria when in association with spider mites [67].

The effects of the host plant or host plant-prey systems on the gut microbiota of $T$. urticae and $N$. californicus did not produce significant differences in the potential functional contribution of their associated microbiota from plant to plant. However, the microbiota associated with each species or stage of development of $T$. urticae had very different potentials to functionally contribute to their hosts. The potential functional contribution of the microbiota associated with $N$. californicus, even by sharing some members of the microbial community with the host, was very distinct. The microbiota of $N$. californicus, particularly the free-living bacteria, provide a significant contribution to host nutritional metabolism (protein digestion and absorption, fat digestion and absorption, mineral absorption, cholesterol metabolism, carotenoid biosynthesis) and defense against pathogenic bacteria and fungi by contributing 
to the production of bioactive molecules (penicillin and cephalosporin biosynthesis, staurosporine biosynthesis, and biosynthesis of various secondary metabolites).

In conclusion, we demonstrated that the microbiota associated with eggs and adult females of $T$. urticae are quite different one from another, and that the microbiota of the predator $N$. californicus does not resemble the microbiota of the prey. We also demonstrated that the microbiota of $T$. urticae is little affected by the host plant, and the microbiota of $N$. californicus has little impact on the host plant-prey system used, both characterized by a discrete dysbiosis effect. Our data also indicated that Rickettsia is a primary symbiont of $T$. urticae, and that both $T$. urticae and $N$. californicus carry potentially pathogenic Pseudomonas species. We also demonstrated that the microbiota of $N$. californicus has a much higher potential to contribute secondary molecules that would protect the host against pathogenic bacteria and fungi than the microbiota associated with T. urticae. The association of Wolbachia with T. urticae and N. californicus was severely affected by the host plant. We also concluded that host plants can induce dysbiosis in the microbiota associated with T. urticae and N. californicus, but changes observed in the microbiota of these mite species from plant to plant did not produce changes in the functional contribution of the associated microbiota, demonstrating the occurrence of redundancy in the functional contributions of the observed dysbiotic microbiota.

\section{Declarations}

\section{Funding}

We thank the Fundação de Amparo à Pesquisa do Estado de São Paulo (FAPESP) for the scholarship granted to BLM (Grant 2018/24768-9). This study is part of a BIOTA/FAPESP project (Process 2017/12004-1).

\section{Conflicts of interest/competing interests}

The authors declare that the research was conducted in the absence of any commercial or financial relationships that could be construed as a potential conflict of interest.

\section{Authors' contributions}

BLM: conceptualization, methods, formal analysis, writing, original draft. GJM: experimental design, writing, revision. FLC: conceptualization, writing, revision and editing, project administration, funding acquisition, supervision.

\section{Availability of data and material}

Not applicable.

\section{Ethical approval}

The article does not contain any human and animal rights. 


\section{Consent to participate/publish}

All authors agreed with their authorship, the final version of the manuscript and with its publication.

\section{Acknowledgements}

We thank the Fundação de Amparo à Pesquisa do Estado de São Paulo (FAPESP) for the scholarship granted to BLM (Grant 2018/24768-9). This study is part of a BIOTA/FAPESP project (Process 2017/12004-1).

\section{Sequence data}

The raw reads obtained by Illumina MiSeq sequencing were deposited in the Sequence Read Archive (SRA) at the National Center for Biotechnology Information under the accession number PRJNA784594.

\section{References}

1. Brinker P, Fontaine MC, Beukeboom LW, Salles JF (2019) Host, symbionts, and the microbiome: the missing tripartite interaction. Trends Microbiol 27:480-488.

https://doi.org/10.1016/j.tim.2019.02.002

2. Cotter SC, Pincheira-Donoso D, Thorogood R (2019) Defences against brood parasites from a social immunity perspective. Philos Trans R Soc B 374:20180207. https://doi.org/10.1098/rstb.2018.0207

3. Douglas AE (2009) The microbial dimension in insect nutritional ecology. Funct Ecol 23:38-47. https://doi.org/10.1111/j.1365-2435.2008.01442.x

4. Leftwich PT, Edgington MP, Chapman T (2020) Transmission efficiency drives host-microbe associations. Proc R Soc B 287:20200820. https://doi.org/10.1098/rspb.2020.0820

5. Fisher RM, Henry LM, Cornwallis CK et al (2017) The evolution of host-symbiont dependence. Nat Commun 8:15973. https://doi.org/10.1038/ncomms15973

6. Van Ham RCHJ, Kamerbeek J, Palacios C et al (2003) Reductive genome evolution in Buchnera aphidicola. Proc Natl Acad Sci USA 100:581-586. https://doi.org/10.1073/pnas.0235981100

7. Ma W-J, Vavre F, Beukeboom LW (2014) Manipulation of arthropod sex determination by endosymbionts: diversity and molecular mechanisms. Sex Dev 8:59-73. https://doi.org/10.1159/000357024

8. Feldhaar H (2011) Bacterial symbionts as mediators of ecologically important traits of insect hosts. Ecol Entomol 36:533-543. https://doi.org/https://doi.org/10.1111/j.1365-2311.2011.01318.x

9. Zhang X-F, Zhao D-X, Li H-S, Hong X-Y (2012) Expression of cytoplasmic incompatibility and host fitness effects in field populations of Sogatella furcifera infected with Cardinium. J Econ Entomol 105:2161-2166. https://doi.org/10.1603/EC12268

10. Schausberger P, Gotoh T, Sato Y (2019) Spider mite mothers adjust reproduction and sons' alternative reproductive tactics to immigrating alien conspecifics. R Soc open Sci 6:191201. 
https://doi.org/10.1098/rsos.191201

11. Santos-Garcia D, Mestre-Rincon N, Zchori-Fein E, Morin S (2020) Inside out: microbiota dynamics during host-plant adaptation of whiteflies. ISME J 14:847-856. https://doi.org/10.1038/s41396-0190576-8

12. Hansen AK, Moran NA (2014) The impact of microbial symbionts on host plant utilization by herbivorous insects. Mol Ecol 23:1473-1496. https://doi.org/10.1111/mec.12421

13. Kikuchi Y, Hayatsu M, Hosokawa T et al (2012) Symbiont-mediated insecticide resistance. Proc Natl Acad Sci USA 109:8618-8622. https://doi.org/10.1073/pnas.1200231109

14. Almeida LG de, Moraes LAB de, Trigo JR et al (2017) The gut microbiota of insecticide-resistant insects houses insecticide-degrading bacteria: A potential source for biotechnological exploitation. PLoS One 12:e0174754. https://doi.org/10.1371/journal.pone.0174754

15. Gomes AFF, Omoto C, Cônsoli FL (2020) Gut bacteria of field-collected larvae of Spodoptera frugiperda undergo selection and are more diverse and active in metabolizing multiple insecticides than laboratory-selected resistant strains. J Pest Sci (2004) 93:833-851. https://doi.org/10.1007/s10340-020-01202-0

16. Chu C-C, Spencer JL, Curzi MJ et al (2013) Gut bacteria facilitate adaptation to crop rotation in the western corn rootworm. Proc Natl Acad Sci USA 110:11917-11922. https://doi.org/10.1073/pnas.1301886110

17. Yun J-H, Roh SW, Whon TW et al (2014) Insect gut bacterial diversity determined by environmental habitat, diet, developmental stage, and phylogeny of host. Appl Environ Microbiol 80:5254. https://doi.org/10.1128/AEM.01226-14

18. Gora N V, Serga S V, Maistrenko OM et al (2020) Climate factors and Wolbachia infection frequencies in natural populations of Drosophila melanogaster. Cytol Genet 54:189-198. https://doi.org/10.3103/S0095452720030044

19. Brady CM, White JA (2013) Cowpea aphid (Aphis craccivora) associated with different host plants has different facultative endosymbionts. Ecol Entomol 38:433-437. https://doi.org/10.1111/een.12020

20. Näsvall K, Wiklund C, Mrazek V et al (2021) Host plant diet affects growth and induces altered gene expression and microbiome composition in the wood white (Leptidea sinapis) butterfly. Mol Ecol 30:499-516. https://doi.org/10.1111/mec.15745

21. Ospina OE, Massey SE, Verle Rodrigues JC (2016) Reduced diversity in the bacteriome of the phytophagous mite Brevipalpus yothersi (Acari: Tenuipalpidae). Insects 7:80. https://doi.org/10.3390/insects7040080

22. Sharon G, Segal D, Ringo JM et al (2010) Commensal bacteria play a role in mating preference of Drosophila melanogaster. Proc Natl Acad Sci USA 107:20051-20056. https://doi.org/10.1073/pnas.1009906107

23. Brucker RM, Bordenstein SR (2013) The hologenomic basis of speciation: Gut bacteria cause hybrid lethality in the genus Nasonia. Science 341:667-669. https://doi.org/10.1126/science.1240659 
24. Pekas A, Palevsky E, Sumner JC et al (2017) Comparison of bacterial microbiota of the predatory mite Neoseiulus cucumeris (Acari: Phytoseiidae) and its factitious prey Tyrophagus putrescentiae (Acari: Acaridae). Sci Rep 7:1-12. https://doi.org/10.1038/s41598-017-00046-6

25. Gols R, Schütte C, Stouthamer R, Dicke M (2007) PCR-based identification of the pathogenic bacterium, Acaricomes phytoseiuli, in the biological control agent Phytoseiulus persimilis (Acari: Phytoseiidae). Biol Control 42:316-325. https://doi.org/10.1016/j.biocontrol.2007.06.001

26. Tinker KA, Ottesen EA (2018) The hindgut microbiota of praying mantids is highly variable and includes both prey-associated and host-specific microbes. PLoS One 13:e0208917. https://doi.org/10.1371/journal.pone.0208917

27. Sugawara R, Ullah MS, Ho C-C, Gotoh T (2018) Impact of temperature-mediated functional responses of Neoseiulus womersleyi and N. longispinosus (Acari: Phytoseiidae) on Tetranychus urticae (Acari: Tetranychidae). Biol Control 126:26-35. https://doi.org/10.1016/j.biocontrol.2018.07.010

28. McMurtry JA, Moraes GJ De, Sourassou NF (2013) Revision of the lifestyles of phytoseiid mites (Acari: Phytoseiidae) and implications for biological control strategies. Syst Appl Acarol 18:297-320. https://doi.org/10.11158/saa.18.4.1

29. Gilbert MTP, Moore W, Melchior L, Worobey M (2007) DNA extraction from dry museum beetles without conferring external morphological damage. PLoS One 2:e272. https://doi.org/10.1371/journal.pone.0000272

30. Sambrook J, Russell DW (2001) Molecular cloning: A laboratory manual. Cold Spring Harbor Laboratory Press, Cold Spring Harbor, NY.

31. Callahan BJ, McMurdie PJ, Rosen MJ et al (2016) DADA2: High-resolution sample inference from Illumina amplicon data. Nat Methods 13:581-583. https://doi.org/10.1038/nmeth.3869

32. R Core Team (2021) R: A language and environment for statistical computing, v.4.0.3. Found Stat Comput, Vienna, Austria. https://www.R-project.org/.

33. Quast C, Pruesse E, Yilmaz P et al (2013) The SILVA ribosomal RNA gene database project: improved data processing and web-based tools. Nucleic Acids Res 41:D590-D596. https://doi.org/10.1093/nar/gks1219

34. Callahan BJ, McMurdie PJ, Holmes SP (2017) Exact sequence variants should replace operational taxonomic units in marker-gene data analysis. ISME J 11:2639-2643. https://doi.org/10.1038/ismej.2017.119

35. Shannon CE (1948) A mathematical theory of communication. Bell Syst Tech J 27:379-423. https://doi.org/10.1002/j.1538-7305.1948.tb01338.x

36. Jaccard $P$ (1908) Nouvelles recherches sur la distribution florale. Bull Soc Vaud Sci Nat 44:223-270

37. Lozupone CA, Hamady M, Scott KT, Knight R (2007) Quantitative and qualitative $\beta$ diversity measures lead to different insights into factors that structure microbial communities. Appl Environ Microbiol 73:1576-1585. https://doi.org/10.1128/AEM.01996-06

38. Lozupone C, Knight R (2005) UniFrac: a New Phylogenetic Method for Comparing Microbial Communities. Appl Environ Microbiol 71:8228-8235. https://doi.org/10.1128/AEM.71.12.8228- 
8235.2005

39. Chong J, Liu P, Zhou G, Xia J (2020) Using MicrobiomeAnalyst for comprehensive statistical, functional, and meta-analysis of microbiome data. Nat Protoc 15:799-821. https://doi.org/10.1038/s41596-019-0264-1

40. Dhariwal A, Chong J, Habib S et al (2017) MicrobiomeAnalyst: a web-based tool for comprehensive statistical, visual and meta-analysis of microbiome data. Nucleic Acids Res 45:W180-W188. https://doi.org/10.1093/nar/gkx295

41. Robinson MD, McCarthy DJ, Smyth GK (2010) edgeR: a Bioconductor package for differential expression analysis of digital gene expression data. Bioinformatics 26:139-140. https://doi.org/10.1093/bioinformatics/btp616

42. Segata N, Izard J, Waldron L et al (2011) Metagenomic biomarker discovery and explanation. Genome Biol 12:1-18. https://doi.org/10.1186/gb-2011-12-6-r60

43. Iwai S, Weinmaier T, Schmidt BL et al (2016) Piphillin: improved prediction of metagenomic content by direct inference from human microbiomes. PLoS One 11:e0166104. https://doi.org/10.1371/journal.pone.0166104

44. Narayan NR, Weinmaier T, Laserna-Mendieta EJ et al (2020) Piphillin predicts metagenomic composition and dynamics from DADA2-corrected 16S rDNA sequences. BMC Genomics 21:1-12. https://doi.org/10.1186/s12864-019-6427-1

45. Koosha M, Vatandoost H, Karimian F et al (2019) Effect of Serratia AS1 (Enterobacteriaceae: Enterobacteriales) on the fitness of Culex pipiens (Diptera: Culicidae) for paratransgenic and RNAi approaches. J Med Entomol 56:553-559. https://doi.org/10.1093/jme/tjy183

46. Perreau J, Patel DJ, Anderson H et al (2021) Vertical transmission at the pathogen-symbiont interface: Serratia symbiotica and aphids. MBio 12:e00359-21. https://doi.org/10.1128/mBio.00359-21

47. Kobiałka M, Michalik A, Świerczewski D, Szklarzewicz T (2019) Complex symbiotic systems of two treehopper species: Centrotus cornutus (Linnaeus, 1758) and Gargara genistae (Fabricius, 1775) (Hemiptera: Cicadomorpha: Membracoidea: Membracidae). Protoplasma 257:819-831. https://doi.org/10.1007/s00709-019-01466-z

48. De Vleesschauwer D, Höfte M (2003) Using Serratia plymuthica to control fungal pathogens of plants. CAB Rev 2:046. https://doi.org/10.1079/PAVSNNR20072046

49. Crooker AR (2000) A Rickettsia in the ovary of the twospotted spider mite, Tetranychus urticae Koch (Acarina: Tetranychedae). Microsc Microanal 6:662-663. https://doi.org/10.1017/S1431927600035807

50. Zhao D-X, Zhang X-F, Chen D-S et al (2013) Wolbachia-host interactions: host mating patterns affect Wolbachia density dynamics. PLoS One 8:e66373. https://doi.org/10.1371/journal.pone.0066373

51. Zhu Y-X, Song Y-L, Hoffmann AA et al (2019) A change in the bacterial community of spider mites decreases fecundity on multiple host plants. MicrobiologyOpen 8:e00743. https://doi.org/10.1002/mbo3.743 
52. Zhu L-Y, Zhang K-J, Zhang Y-K et al (2012) Wolbachia strengthens Cardinium-induced cytoplasmic incompatibility in the spider mite Tetranychus piercei McGregor. Curr Microbiol 65:516-523. https://doi.org/10.1007/s00284-012-0190-8

53. Swei A, Kwan JY (2017) Tick microbiome and pathogen acquisition altered by host blood meal. ISME J 11:813-816. https://doi.org/10.1038/ismej.2016.152

54. Giorgini M, Bernardo U, Monti MM et al (2010) Rickettsia symbionts cause parthenogenetic reproduction in the parasitoid wasp Pnigalio soemius (Hymenoptera: Eulophidae). Appl Environ Microbiol 76:2589. https://doi.org/10.1128/AEM.03154-09

55. Brumin M, Kontsedalov S, Ghanim M (2011) Rickettsia influences thermotolerance in the whitefly Bemisia tabaci B biotype. Insect Sci 18:57-66. https://doi.org/10.1111/j.1744-7917.2010.01396.x

56. Hunter DJ, Torkelson JL, Bodnar J et al (2015) The Rickettsia endosymbiont of Ixodes pacificus contains all the genes of de novo folate biosynthesis. PLoS One 10:e0144552. https://doi.org/10.1371/journal.pone.0144552

57. Shan H, Liu Y, Luan J, Liu S (2021) New insights into the transovarial transmission of the symbiont Rickettsia in whiteflies. Sci China Life Sci 64:1174-1186. https://doi.org/10.1007/s11427-020-18017

58. Caspi-Fluger A, Inbar M, Mozes-Daube $\mathrm{N}$ et al (2012) Horizontal transmission of the insect symbiont Rickettsia is plant-mediated. Proc R Soc B Biol Sci 279:1791-1796. https://doi.org/10.1098/rspb.2011.2095

59. Sourassou NF, Hanna R, Breeuwer JAJ et al (2014) The endosymbionts Wolbachia and Cardinium and their effects in three populations of the predatory mite Neoseiulus paspalivorus. Exp Appl Acarol 64:207-221. https://doi.org/10.1007/s10493-014-9820-0

60. Charlat S, Hurst GDD, Merçot H (2003) Evolutionary consequences of Wolbachia infections. Trends Genet 19:217-223. https://doi.org/10.1016/S0168-9525(03)00024-6

61. Zhu Y-X, Song Y-L, Zhang Y-K et al (2018) Incidence of facultative bacterial endosymbionts in spider mites associated with local environments and host plants. Appl Environ Microbiol 84: e02546-17. https://doi.org/10.1128/AEM.02546-17

62. Zélé F, Santos JL, Godinho DP, Magalhães S (2018) Wolbachia both aids and hampers the performance of spider mites on different host plants. FEMS Microbiol Ecol 94:fiy187. https://doi.org/10.1093/femsec/fiy187

63. Coon KL, Valzania L, Brown MR, Strand MR (2020) Predaceous Toxorhynchites mosquitoes require a living gut microbiota to develop. Proc R Soc B Biol Sci 287:20192705. https://doi.org/10.1098/rspb.2019.2705

64. Dion-Phénix H, Charmantier A, de Franceschi C et al (2021) Bacterial microbiota similarity between predators and prey in a blue tit trophic network. ISME J 15:1098-1107. https://doi.org/10.1038/s41396-020-00836-3

65. Liu G, Zheng X, Long H et al (2021) Gut bacterial and fungal communities of the wild and laboratoryreared Thitarodes larvae, host of the Chinese medicinal fungus Ophiocordyceps sinensis on Tibetan 
plateau. Insects 12:. https://doi.org/10.3390/insects12040327

66. König H (2006) Bacillus species in the intestine of termites and other soil invertebrates. J Appl Microbiol 101:620-627. https://doi.org/10.1111/j.1365-2672.2006.02914.x

67. Zhu Y-X, Song Z-R, Song Y-L et al (2020) The microbiota in spider mite feces potentially reflects intestinal bacterial communities in the host. Insect Sci 27:859-868. https://doi.org/10.1111/17447917.12716

68. Hu Z, Chen X, Chang J et al (2018) Compositional and predicted functional analysis of the gut microbiota of Radix auricularia (Linnaeus) via high-throughput Illumina sequencing. PeerJ 6:e5537. https://doi.org/10.7717/peerj.5537

69. Frigerio J, Agostinetto G, Galimberti A et al (2020) Tasting the differences: Microbiota analysis of different insect-based novel food. Food Res Int 137:109426. https://doi.org/10.1016/j.foodres.2020.109426

70. Mercado-Blanco J, Bakker PAHM (2007) Interactions between plants and beneficial Pseudomonas spp.: exploiting bacterial traits for crop protection. Antonie Van Leeuwenhoek 92:367-389. https://doi.org/10.1007/s10482-007-9167-1

71. Kupferschmied P, Maurhofer M, Keel C (2013) Promise for plant pest control: root-associated pseudomonads with insecticidal activities. Front Plant Sci 4:287. https://doi.org/10.3389/fpls.2013.00287

72. Rajkumar M, Bruno LB, Banu JR (2017) Alleviation of environmental stress in plants: The role of beneficial Pseudomonas spp. Crit Rev Environ Sci Technol 47:372-407. https://doi.org/10.1080/10643389.2017.1318619

73. Aksoy HM, Ozman-Sullivan SK, Ocal H et al (2008) The effects of Pseudomonas putida biotype B on Tetranychus urticae (Acari: Tetranychidae). In: Bruin J, van der Geest LPS (eds) Diseases of mites and ticks. Springer, Dordrecht, pp 223-230. https://doi.org/10.1007/978-1-4020-9695-2_18

74. Quesssaoui R, Bouharroud R, Amarraque A et al (2017) Ecological applications of Pseudomonas as a biopesticide to control two-spotted mite Tetranychus urticae. chitinase and HCN production. J Plant Prot Res 57:409-416. https://doi.org/10.1515/jppr-2017-0055

75. Flury P, Vesga P, Péchy-Tarr M et al (2017) Antimicrobial and insecticidal: Cyclic lipopeptides and hydrogen cyanide produced by plant-beneficial Pseudomonas strains CHA0, CMR12a, and PCL1391 contribute to insect killing. Front Microbiol 8:100. https://doi.org/10.3389/fmicb.2017.00100

76. Friman J, Pineda A, Gershenzon J et al (2021) Differential effects of the rhizobacterium Pseudomonas simiae on above- and belowground chewing insect herbivores. J Appl Entomol 145:250-260. https://doi.org/10.1111/jen.12842

77. Kupferschmied P, Chai T, Flury P et al (2016) Specific surface glycan decorations enable antimicrobial peptide resistance in plant-beneficial pseudomonads with insect-pathogenic properties. Environ Microbiol 18:4265-4281. https://doi.org/10.1111/1462-2920.13571

78. Vacheron J, Péchy-Tarr M, Brochet S et al (2019) T6SS contributes to gut microbiome invasion and killing of an herbivorous pest insect by plant-beneficial Pseudomonas protegens. ISME J 13:1318- 
1329. https://doi.org/10.1038/s41396-019-0353-8

79. Erban T, Rybanska D, Harant K et al (2016) Feces derived allergens of Tyrophagus putrescentiae reared on dried dog food and evidence of the strong nutritional interaction between the mite and Bacillus cereus producing protease bacillolysins and exo-chitinases. Front Physiol 7:53. https://doi.org/10.3389/fphys.2016.00053

\section{Figures}

\section{Figure 1}

Relative abundance of the bacterial taxon hits at the (A) class and (B) genus taxonomic levels in the microbiota associated with eggs and adult females of Tetranychus urticae, and with adult females of the predatory mite Neoseiulus californicus reared on cotton, maize, pinto bean, and tomato as host plants

\section{Figure 2}

Shannon indexes of the bacterial communities associated with eggs and adult females of Tetranychus urticae and with adult females of the predatory mite Neoseiulus californicus reared on different host plants

\section{Figure 3}

Principal coordinates analysis ( $P C O A)$ from the distance matrix generated by Jaccard $(A, B, C)$ and weighted $(D, E, F)$ and unweighted $(G, H, I)$ Unifrac indices for comparisons among adult females of Tetranychus urticae $(B, D, G)$, eggs of $T$. urticae $(B, E, H)$, and adult females of Neoseiulus californicus (C, F, I) from different host plants

\section{Figure 4}

Heatmaps clustering taxonomic abundance at ASV (A), class (B), and genus (C) levels in samples of eggs and adult females of Tetranychus urticae and of adult females of the predatory mite Neoseiulus californicus reared on different host plants

\section{Figure 5}


Dendrogram produced using the distances based on weighted Unifrac for microbiota associated with (A) eggs and adult females of Tetranychus urticae and (B) eggs of T. urticae and their predator Neoseiulus californicus reared on different host plants

\section{Figure 6}

Box plot representation for genera that showed significant differences in abundance between the microbiota of eggs of Tetranychus urticae and females of Neoseiulus californicus reared on maize, pinto bean, and tomato leaves

\section{Figure 7}

Box-plots representation for genera that showed significant differences in abundance between the microbiota of eggs and adult females of Tetranychus urticae reared on cotton, maize, pinto bean, and tomato leaves

\section{Figure 8}

LEfSe analysis of the bacterial biomarkers associated with eggs and adult females of Tetranychus urticae and with adult females of Neoseiulus californicus. Cladogram represents the hierarchical taxonomic structure from phylum to genus of the biomarkers identified

\section{Figure 9}

Relative abundances of the metabolic pathways represented in eggs and adult females of Tetranychus urticae and in adult females of Neoseiulus californicus from cotton, maize, pinto bean, and tomato host plants

\section{Figure 10}

Potential functional contributions of the microbiota associated with eggs and adult females of Tetranychus urticae and with the predatory mite Neoseiulus californicus in several pathways when mites are maintained on different host plants

\section{Supplementary Files}

This is a list of supplementary files associated with this preprint. Click to download. 
- BLMmanuscriptmetabarcodingsupplementarymaterial.docx 